\title{
Characterization of Thermomechanical Damage on Tungsten Surfaces During Long-Duration Plasma Transients
}

\author{
David Rivera, Tamer Crosby, Andrew Sheng, Nasr M. Ghoniem \\ Department of Mechanical \& Aerospace Engineering, University of California at Los Angeles (UCLA), 420 \\ Westwood Plaza, Los Angeles, CA. 90095-1597, U.S.A. \\ david.rivera.ucla@gmail.com
}

\begin{abstract}
A new experimental facility constructed at UCLA for the simulation of high heat flux effects on plasma-facing materials is described. The High Energy Flux Test Facility (HEFTY) is equipped with a Praxair model SG-100 plasma gun, which is nominally rated at $80 \mathrm{~kW}$ of continuous operation, of which approximately $30 \mathrm{~kW}$ reaches the target due to thermal losses. The gun is used to impart high intermittent heat flux to metal samples mounted within a cylindrical chamber. The system is capable of delivering an instantaneous heat flux in the range of $30-300 \mathrm{MW} / \mathrm{m}^{2}$, depending on sample proximity to the gun. The duration of the plasma heat flux is in the range of 1-1000 s, making it ideal for studies of mild plasma transients of relatively long duration. Tungsten and tungsten-copper alloy metal samples are tested in these transient heat flux conditions, and the surface is characterized for damage evaluation using optical, SEM, XRD, and micro-fabrication techniques. Results from a Finite Element (FE) thermo-elastoplasticity model indicate that during the heat-up phase of a plasma transient pulse, the majority of the sample surface is under compressive stresses leading to plastic deformation of the surface. Upon sample cooling, the recovered elastic strain of cooler parts of the sample exceeds that from parts that deformed plastically, resulting is a tensile surface self-stress (residual surface stress). The intensity of the residual tensile surface stress is experimentally correlated with the onset of complex surface fracture morphology on the tungsten surface, and extending below the surface region. Micro-compression mechanical tests of $\mathrm{W}$ micro-pillars show that the material has significant plasticity, failing by a "barreling"mode before plasma exposure, and by normal dislocation slip and localized shear after plasma exposure. Ongoing modeling of the complex thermo-fracture process, coupled with elasto-plasticity is based on a phase field approach for distributed fracture, and a discrete cracking approach, with cracks represented by Volterra dislocations.
\end{abstract}

Keywords: tungsten, phase field, cracks, thermomechanics, plasma transients, surface fracture.

\section{Introduction}

Severe plasma transients are expected to greatly influence the integrity of plasma-facing components in fusion energy systems, notably the tungsten armor in most of existing divertor designs [1]. A similar technological application is encountered in Space Electric Propulsion (EP) and Pulsed Power (PP) devices that utilize plasma and ion thrusters for space and military applications. Since tungsten is one of the highest melting point materials, it is proposed as a plasma facing component for these severe environments. Two main aspects of damage degrade 
the performance of tungsten during plasma transients, the first of which is thermomechanical in origin, while the second is due to the slow accumulation of plasma gases (e.g. helium and hydrogen bubbles)- underneath the surface layer. Typical design-base transients contain an amount of energy in between 0.1 and $0.5 \mathrm{MJ} / \mathrm{m}^{2}$ for the Joint European Torus (JET) and between 1 and $5 \mathrm{MJ} / \mathrm{m}^{2}$ for ITER, lasting a duration of $0.1-1 \mathrm{~ms}$. Such transients are expected to cause surface fracture, plasticity, grain motion in polycrystalline tungsten. Because plasma transient times are much shorter than the thermal diffusion time in a typical $3 \mathrm{~mm}$ cooled $\mathrm{W}$ plate (on the order of $0.25 \mathrm{~s}$ ), high energy densities cannot be totally dissipated in tungsten surface layers, and thus, to first order, damage phenomena will be controlled by the energy density rather than the energy flux density. Among the operational scenarios of interest is the response of tungsten to Edge Localized Modes, which carry energy densities on the order of $0.1-5 \mathrm{MJ} / \mathrm{m}^{2}$ and time durations in the range of $0.1-0.5 \mathrm{~ms}$, which can result in severe damage to the tungsten armor in the form of cracking, localized melting, and evaporation[2]. The challenges facing tungsten as a plasma-facing component (PFC) candidate material are further exacerbated upon consideration of the pulsed nature of the incoming heat flux. Bazylev et al. carried out experiments in the plasma gun facilities QSPA-T for the ITER-like ELM heat loads, and showed significant erosion of tungsten macro brush edges. Numerical simulations of the target damage were carried out for QSPA-T conditions using the code PHEMOBRID [3].

In the Heat and Nuclear Load Specifications (HNLS) document, which is one of the ITER Baseline design documents, several types of plasma transients are described [4]. These are: (1) Major plasma disruptions as a result of reaching global stability limits in beta or the plasma density (MHD mode), (2) Vertical Displacement Events (VDE) as a result of loss of plasma vertical position, (3) Edge Localized Modes (ELM), of type I, II, and III, (4) Overlapping ELM-filament heat excursions, and (5) Plasma power startup and shut-down transients, where the fusion power reaches quasi-steady state in about $100 \mathrm{~s}$. The first two categories of transients, as well as ELMtype I will have energy densities in excess of $1 \mathrm{MJ} / \mathrm{m}^{2}$, causing significant melting and evaporation of W Plasma Facing Components (PFCs) [4]. Regardless of the cooling technology, no material can withstand these severe plasma events without melting and evaporation [5]. They are thus excluded from the normal operational design basis, and a Disruption Mitigation System (DMS) must be designed to handle the excessive heat loads in such short transients. DMS considerations are being developed using massive gas injection [4]. The fourth and fifth category of transients can be much slower, although some uncertainties remain in understanding the propagation of ELM filaments through the Scrape-Off Layer (SOL). Uncontrolled ELM filaments can propagate at $1 \mathrm{~km} / \mathrm{s}$ [6], while controlled ELMs would propagate at a slower rate. There is a significant probability that a series of subsequent filaments from different ELSs will overlap and produce a heat flux fluctuation over a timescale of the order of $0.5 \mathrm{~s}$ [4]. Thermomechanical damage phenomena associated with startup/ shutdown plasma transients, and with overlapping controlled ELMs are expected to be within the design basis, since melting and evaporation do not occur, and limited cracking and plasticity take place and accumulate from cycle-to-cycle.

The forgoing discussion indicates that severe plasma transients with short deposition times (below $1 \mathrm{~ms}$ ) and energy density above $0.5 \mathrm{MJ} / \mathrm{m}^{2}$ should be mitigated, and cannot be considered as a design basis. However, it is clear that PFCs will experience a wide range of plasma transients during the component lifetime. PFCs in fusion devices must thus be capable of withstanding multiple heat flux cycles of a variety of amplitudes and durations, which in turn lead to a unique thermo-mechanical fatigue environment [7] that is not seen in any design application 
outside of fusion. The damage characteristics of tungsten under intermittent and pulsed high heat flux conditions is thus an important problem to resolve if the material is to be used in for fusion energy applications. A number of recent experimental and modeling efforts have been developed, or are currently under development, utilizing powerful quasi-steady-state plasma accelerators for simulation of ITER transient heat loads on divertor surfaces [8].

In an effort to further understand the physical mechanisms which govern the damage evolution behavior of tungsten in a high heat flux environment, a series of experiments have been conducted in which pure tungsten specimens are exposed to many high heat flux cycles. After repeated exposure to high heat flux pulses, micro-pillars are fabricated on the surface of the irradiated tungsten and the mechanical properties of the material are observed as a function of heat flux exposure. Experimental characterization of ensuing surface damage is presented in Section 2, where the main plasma exposure, high heat flux facility at UCLA (HEFTY), is described in Subsection 2.1. Material characterization is given in Subsection 2.2 for results on micro-compression tests, and Subsection 2.3, for SEM and microscopy test results. To analyze the results of experimental tests, the origin of thermomechanical damage is modeled in Section 3, where a Finite Element thermo-elasto-plasticity model is first presented in Subsection 3.1, followed by a discussion of the ensuing fracture model in Subsection cracking. Finally, conclusions of the study are presented in Section 4.

\section{Experimental Characterization of Surface Damage}

\subsection{Thermomechanical Plasma Testing Facility}

A dedicated system for high heat flux testing has been designed and built to carry out thermomechanical damage characterization experiments. The HEFTY testing facility at UCLA consists of a Praxair SG-100 plasma gun (arc-jet), which is mounted onto the end of a plasma chamber and is used solely as a heat source. The magnitude and duration of the heat flux imparted to samples is computer controlled, and the number of pulses is also programmable. Circular tungsten specimens of $5.08 \mathrm{~cm}$ diameter and $0.508 \mathrm{~mm}$ thickness are mechanically attached to a specially designed sample holder, which delivers a constant jet of impinging cooling water (at room temperature) onto the back surface of the samples, while the front surface remains exposed to the incoming plasma heat flux as seen in figure 1. Calculated convective heat transfer coefficients on the order of $10^{6} \mathrm{~W} / \mathrm{m}^{2} \mathrm{~K}$ have been obtained for the back surface of the samples for a typical flow rate of $19 \mathrm{~L} / \mathrm{min}$. This flow rate can be reduced to reproduce a variety of experimental conditions. The aim of testing materials under cooling is primarily to generate thermal gradients in thick specimens, which in turn, will result in a range of thermal stress states that can be controlled by varying the cooling and heating rates of the samples. The dimensions of test chamber are: length $=81.28 \mathrm{~cm}$, and diameter $=60.33 \mathrm{~cm}$, operating at atmospheric pressure, and uses Ar as a shroud gas to guard against oxidation of the sample surface. The installed diagnostics consist of thermocouples, pressure gauges, and flow meters to determine quantities such as the energy absorbed by the cooling circuit during an experimental run, as well as to ensure that the experimental conditions are within the safety confines of the unit. Other diagnostics include a dedicated data acquisition and computer control system, voltage and current meters, and a LabView interface. 


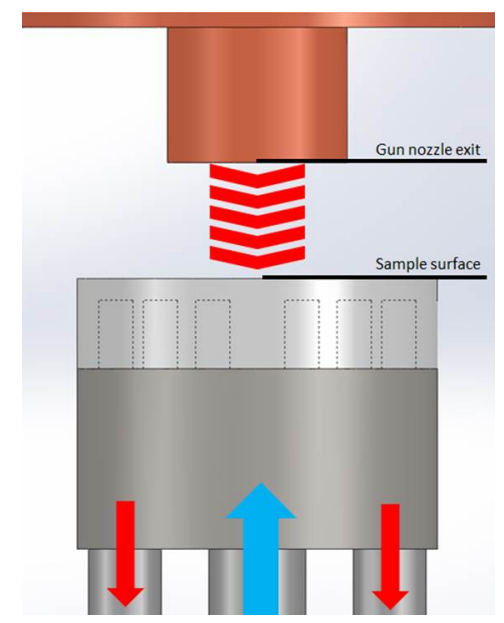

Figure 1: Illustration of a top view of sample holder assembly, specimens are mounted onto a specialized flange which provides constant impingement cooling during testing as described in the text, blue and red arrows indicate the direction of incoming and outgoing water flow respectively, while the red chevrons indicate the incoming heat flux striking the sample surface.

\subsubsection{Heat Flux Measurement}

Two independent methods of heat flux measurements are typically performed. The first is an inverse method, where a transient FEM model is set up with an unknown impinging heat flux. Temperature measurements at the back side of the sample are used to calibrate the model and determine the unknown heat flux. Another method is through calorimetry, where the inlet and exit temperatures of the cooling water to the gun are measured, and knowing the flow rate of the cooling water, we determine the energy absorbed in each pulse. Since the pulse duration is known, this results in an independent check for the heat flux determined by the inverse method. The heat flux seen in this experiment was calculated through the use of a specially designed heat flux sensor similar to a null point calorimeter. Experimental temperature vs. time profiles were generated at specified distances from the gun nozzle exit as seen in figure 1 and the resulting data matched with calculated heat flux values from a finite element model of the sensor. The heat flux values seen in this experiment are all measured at a distance of $1.9 \mathrm{~cm}$ from the nozzle exit and are calculated to be $\sim 25 \mathrm{MW} / \mathrm{m}^{2}$.

\subsubsection{Experimental Conditions}

The plasma stream consists of an Argon gas at atmospheric pressure, with a measured heat flux of $25 \mathrm{MW} / \mathrm{m}^{2}$ when the sample surface is at a distance of $1.9 \mathrm{~cm}$ from the gun nozzle exit as seen in figure 1 . The spot size of the plasma stream striking the surface of the samples was on the order of $1 \mathrm{~cm}$ based on the nozzle diameter and distance from the gun. The Ar flow rate through the gun was kept at $80-100 \mathrm{~L} / \mathrm{min}$ throughout the duration of the experiment. Ar was also used as a shroud gas to prevent oxidation of the sample surface at high temperatures.

While the measured heat flux is relatively low compared with previous experimental simulations of more intense plasma transients in excess of $100 \mathrm{MW} / \mathrm{m}^{2}[9,10]$, it allows for studies of the influence of low intensity and long-duration plasma transients that are also expected in fusion, EP, and PP devices. The samples tested in this experimental campaign were initially polished down to a 400 grit griding paper to prevent premature cracking from rough surface

notches. Samples were subsequently exposed to 1100 pulses of $25 \mathrm{MW} / \mathrm{m}^{2}$ heat flux, each with 


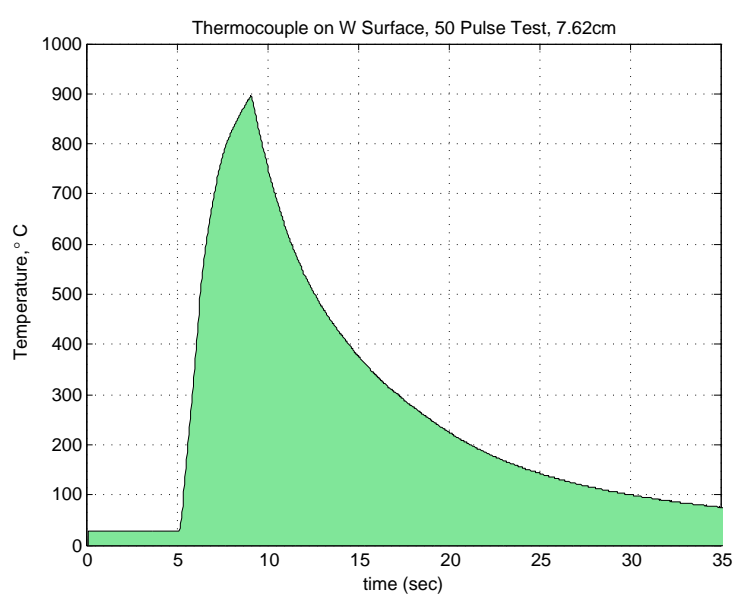

(a)

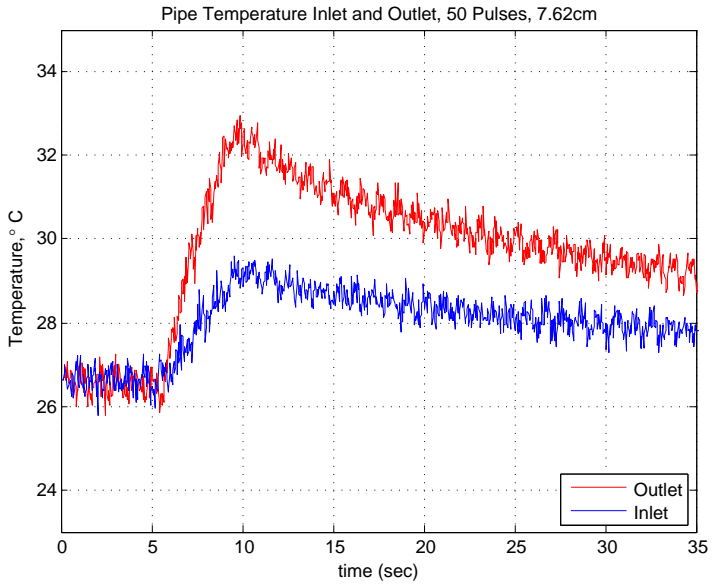

(b)

Figure 2: (a) Zoom in of the temperature profile near the sample surface of a single exposure cycle at $7.62 \mathrm{~cm}$ from the gun nozzle exit. The pulse duration was $4 \mathrm{sec}$, and the sample was allowed to cool for $30 \mathrm{sec}$ in-between pulses to better simulate thermal shock conditions, (b) temperature profile for thermocouples located in the inlet and outlet pipes of the sample holder for a test conducted $7.62 \mathrm{c} \mathrm{m}$ from the gun nozzle exit

a duration of 4-6 seconds. Typical measured temperature profiles near the sample surface (front side facing plasma) are shown in figure 2(a), and can be clearly seen to approach the recrystallization temperature of tungsten $\left(\sim 1300^{\circ} \mathrm{C}\right)$ [11]. The sample holder is designed in such a way that a constant jet of impinging water strikes the back surface of the sample at all times. Three tubes (one inlet and two outlet) carry water to and from the sample. Thermocouples were affixed to the inlet and outlet streams to monitor the change in temperature of the water during each pulse, this data can be seen for a single pulse in figure 2(b).

Because the samples are highly cooled and relatively thin, no extreme local overheating was evident, and the samples did not exhibit evidence of melting or erosion of the surface. Experimental pulsed simulation conditions were selected to examine surface damage solely as a result of thermomechanical loading, while not reaching conditions of melting, and vaporization normally seen in more extreme plasma transients $[2,8]$. As a result of the fact that the facility is primarily concerned with the effects of thermomechanical loading, diagnostics precisely characterizing the plasma are not currently installed. In light of this, an estimate of the incoming ion energies for this particular experimental run can be taken to be on the order of $0.5 \mathrm{eV}$.

The following two subsections describe experimental results for the mechanical (Subsection 2.2), and microstructural (Subsection 2.3) characterization of the influence of plasma transients on commercially pure $\mathrm{W}$.

\subsection{Mechanical Behavior of Tungsten Using Micro-compression Testing}

To characterize the mechanical state of samples exposed to pulsed high heat flux of moderate intensity and long duration, micro-pillars were fabricated on the surface of both pristine and exposed specimens using a FEI Nova600 Nanolab DualBeam FIB-SEM. This mechaniccal testing method has been the subject of recent development, with many applications [12, 13]. For a review of the technique, the reader is referred to the article by Uchic et al. [14]. The height of the pillars was on the order of 10-12 $\mu \mathrm{m}$, with a diameter of $\sim 4 \mu \mathrm{m}$, measured at the 
midsection of the pillar to account for the slight taper introduced as a result of the fabrication process. Stress vs. strain data was subsequently generated upon compression testing. The tests were conducted at room temperature with a constant strain rate of $10^{-3} \mathrm{~s}^{-1}$. SEM images of the pillars can be seen in figure 2.2. Pristine samples exhibited a surprisingly large degree of plastic deformation, possibly a result of the small ratio of pillar size to grain size. This quasi-ductile behavior is not typical of bulk polycrystalline tungsten which exhibits nearly no yield point at room temperature [15].

The average dimensions of individual grains within the pristine pillars are on the order of $0.3 \times 2.5 \mu \mathrm{m}$ in the direction of the applied load, and transverse directions respectively. Average dimensions of the compressed pillars in the loading direction were measured to be $0.18 \mu \mathrm{m}$. This corresponds to a plastic strain of $40 \%$ which is on the same order as that seen in the mechanical testing data. Accurate average grain size in the transverse direction for the compressed pillars proved difficult to collect due the highly textured nature of the material, however, a grain size increase in the transverse direction can be predicted to be on the order of $0.3 \mu \mathrm{m}$ from Poisson effects.

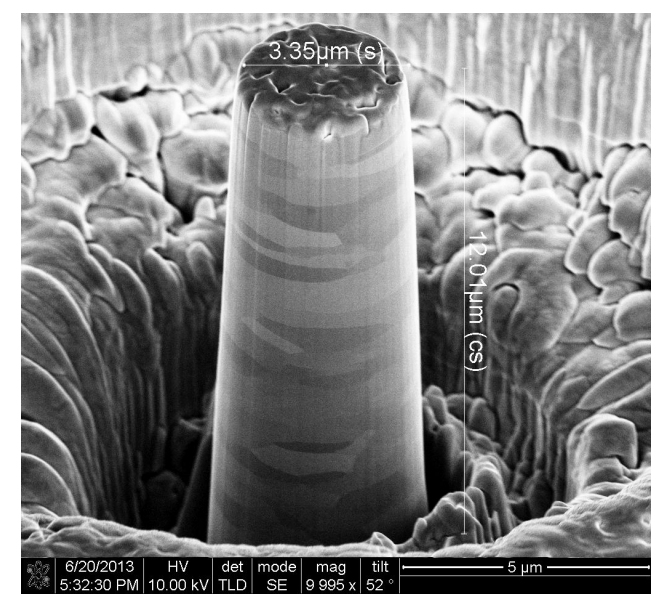

(a)

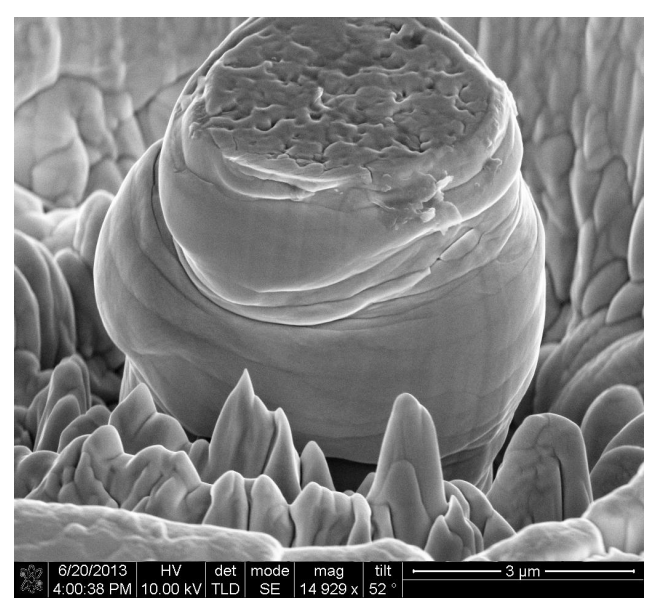

(b)

Figure 3: SEM images of micro-pillars (a) pristine pillar before compression, showing the polycrystalline nature of the material, (b) Compressed pillar (pristine material), note the severe plastic deformation of the column in a "barreling" mode of failure. Pillars seen here are representative of pillars 3 and 4 seen in figure 4

The stress vs. strain data indicate that the tungsten surface progressively softens with increasing exposure to the plasma heat flux, as can be seen in Figure 4. Another feature to note is the change of the plastic region of the stress-strain curves from a linear work hardening mode to a more parabolic work hardening rate after plasma exposure. This may be a result of the onset of dislocation recovery mechanisms upon cyclic heating. Recovery of the dislocation microstructure may explain the reason for the reduction in initial yield stress after exposure, which would correspond with the primary stage of the recovery process.

The combination of recovery and plastic deformation processes occurring simultaneously within the material leads to a state of competition between the two mechanisms. In essence, the material is experiencing anisothermal annealing while being plastically deformed, this prevents it from experiencing the full degree of hardening which would otherwise be seen for an equivalent amount of plastic deformation in the absence of a high temperature environment. Such conditions naturally indicate a reduction in the magnitude of the accumulated net residual stress 


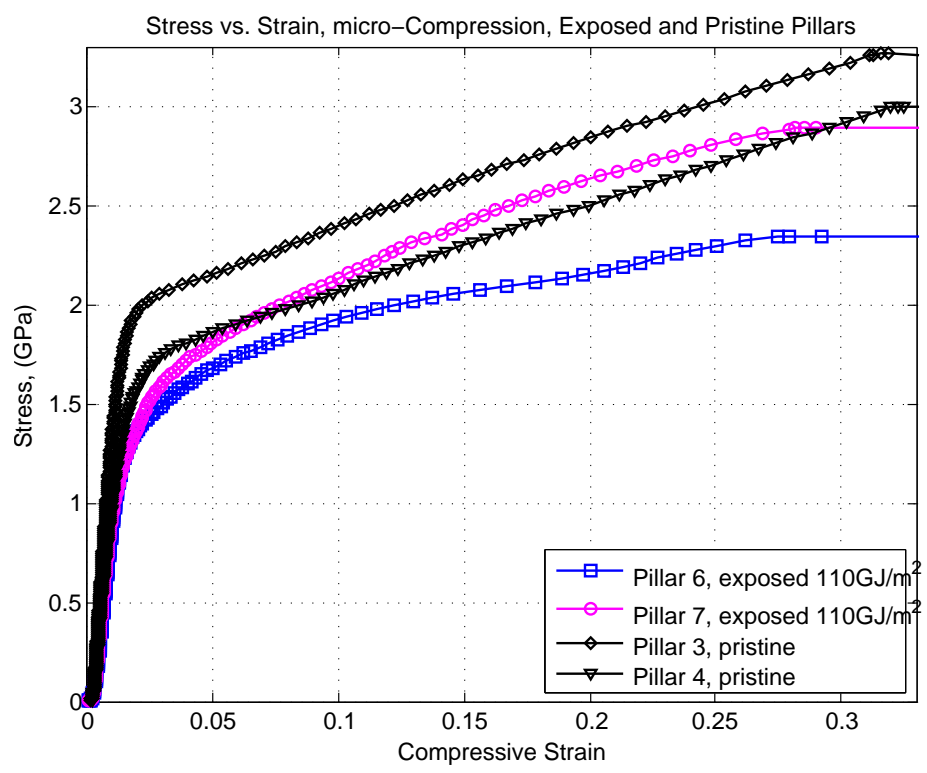

Figure 4: Stress vs. Strain data for exposed and pristine micro-pillars, note the reduction in yield strength, and slight reduction in strain to failure for the exposed specimens. Note that pristine polycrystalline specimens failed at over 30\% compressive strain, buckling is assumed to occur when the plastic portion of the curves reach zero slope, total absorbed energy density calculated to be $25 \mathrm{MW} / \mathrm{m}^{2} \times 1100$ pulses $\times 4 \mathrm{sec}=110 \mathrm{GJ} / \mathrm{m}^{2}$

at the end of every thermal cycle, and thus a prolonged lifetime to failure as the material will require a greater number of cycles to reach a critical residual stress necessary for fracture. Post compression SEM examination of the micro-pillars fabricated from exposed material reveal that the deformation mechanism changed from a barreling mode to a more standard shearing failure as can be seen in figure $5(a)$.

\subsection{Microstructure Characterization}

Microstructure observations of samples exposed to pulsed plasma heat flux included optical, SEM, XRD, and micro-fabrication techniques. While Focused Ion Beam (FIB) techniques were utilized to fabricate samples for mechanical testing, as described in the previous section, Scanning Electron Microscopy (SEM) was subsequently used to examine the samples at intervals of every 100 pulses. The primary features searched for were the onset of cracking or general surface defects induced by mechanical response to the incoming heat flux. Figure 6(a) shows SEM pictures of fractured $\mathrm{W}$ surface after total exposure to $100 \mathrm{GJ} / \mathrm{m}^{2}$ (i.e. 1000 pulses), The complex nature of surface and subsurface fracture is evident. Figure 6(b) indicates that surface fracture results from the tensile loading, following plastic stress relaxation on the cooling side of the pulse, as will be discussed in the modeling section of the paper. These observations are guiding model development, since it is clear that current fracture mechanics approaches are inadequate for description of such complex plasma-induced fracture phenomena, and that alternate and creative approaches are necessary. 


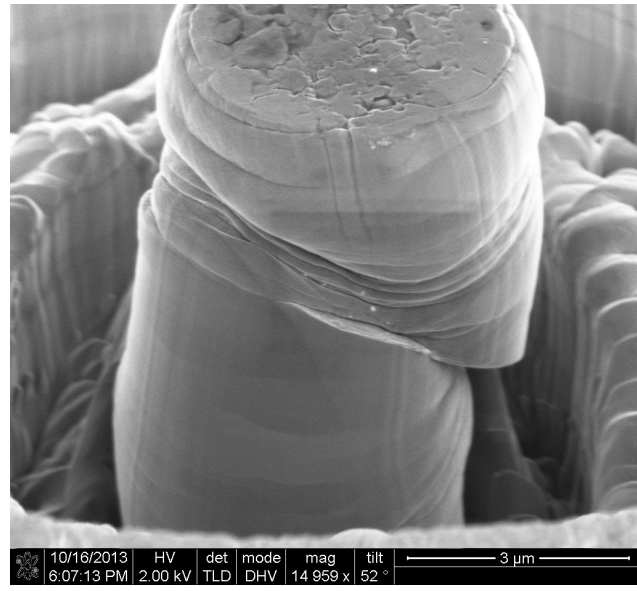

(a)

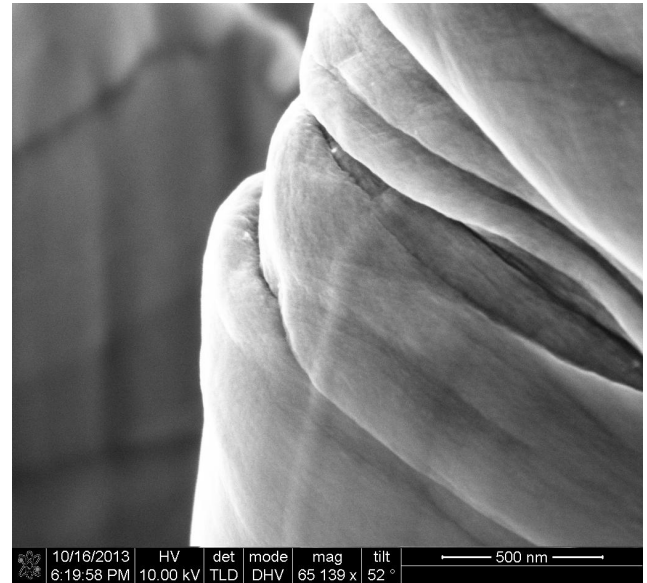

(b)

Figure 5: Deformation mode of micro-pillars fabricated from exposed material, accumulated a total energy density of $110 \mathrm{GJ} / \mathrm{m}^{2}$, (a) pillar exhibited a shearing form of failure and a slight reduction in ductility, (b) Close up view of the flow localization seen in exposed material, slip band formation was prevalent, indicating a micro-structural change absent in the compression of pristine pillars, both images are representative of Pillars 6 and 7 in the stressstrain curves seen in figure 4

\section{Modeling Plasma-Induced Thermomechanical Damage}

\subsection{Finite Element Thermo-Elasto-plasticity Model}

In an effort to better understand the stress and temperature fields of the material, a FEM model of the sample was developed to include the effects of plastic deformation during impingement of the plasma heat flux. The plasticity model considers isotropic hardening with a tangent modulus of $\sim 4.4 \mathrm{GPa}$, and a yield stress of $\sim 1.2 \mathrm{GPa}$ taken from the generated stress vs. strain curves seen in figure 4. Although the material properties of tungsten will invariably depend on temperature, the model incorporates the data generated from the micro-compression testing in an effort to simulate the mechanical response of the samples used in this particular experimental campaign. In this sense, the model conservatively estimates the plastic deformation behavior of the specimens during a transient event. In addition, an applied heat flux of 20 $\mathrm{MW} / \mathrm{m}^{2}$ was used as the plasma facing boundary condition to account for the fact that not all of the incoming heat flux is absorbed by the sample.

The surface plot of the von Mises stress indicates that plasticity in the material is present as seen in figure 7(a). The material reaches the indicated yield stress for tungsten in the central section of the specimen.

It can be observed from the FEM results that the state of stress is initially compressive in the samples and then transitions to a tensile stress upon the cool down phase of the cycle, as seen in figure 8(a). This can be understood by considering that upon the plasma initially striking the cool tungsten surface, the center portion of material directly struck by the plasma attempts to expand upon heating, while the cooler surrounding material constricts the expansion. This behavior results in a state of net compressive stress in the center portion of the samples, which drives plastic deformation under the testing conditions present in the experimental setup. The material then having plastically deformed, undergoes a cool down phase of the cycle. As the plastically deformed material in the center portion of the sample is cooled, it attempts to retract itself back to its equilibrium dimensions at room temperature while the surrounding 


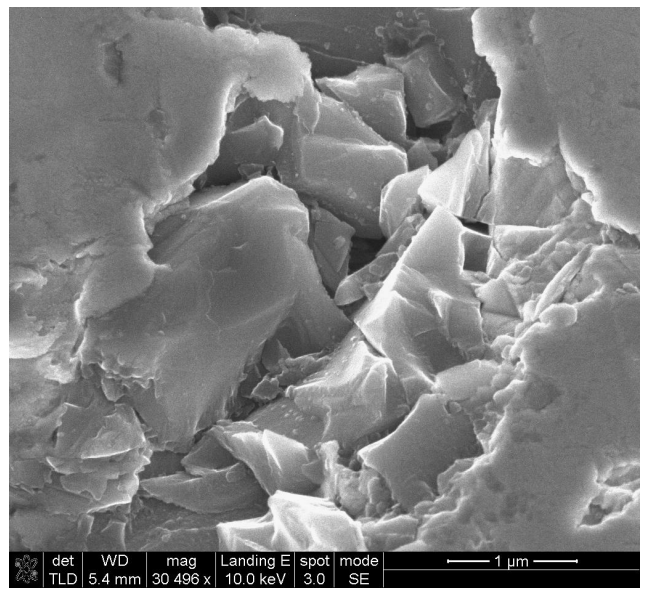

(a)

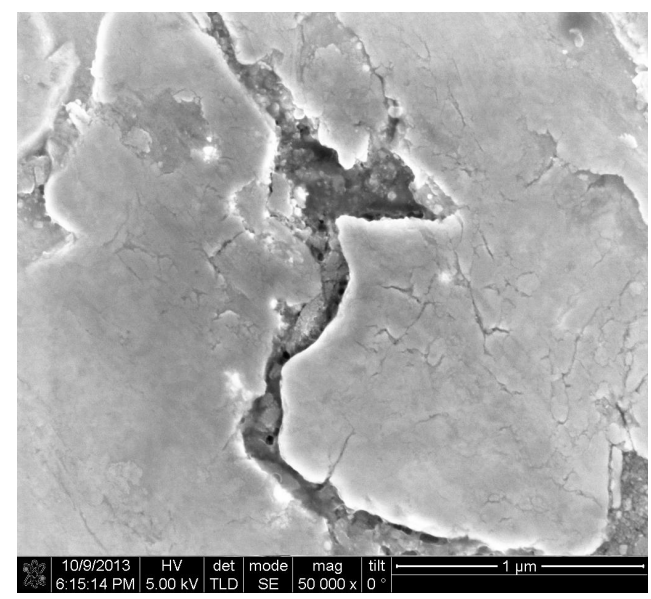

(b)

Figure 6: SEM pictures of fractured $\mathrm{W}$ surface after exposure to one-thousand $25 \mathrm{MW} / \mathrm{m}^{2}$ pulses, each with a duration of $4 \mathrm{~s}$ for a total absorbed energy density of $100 \mathrm{GJ} / \mathrm{m}^{2}$. (a) shows the complex nature of the fracture process, while (b) corroborates the results of elasto-plastic modeling in the next section.

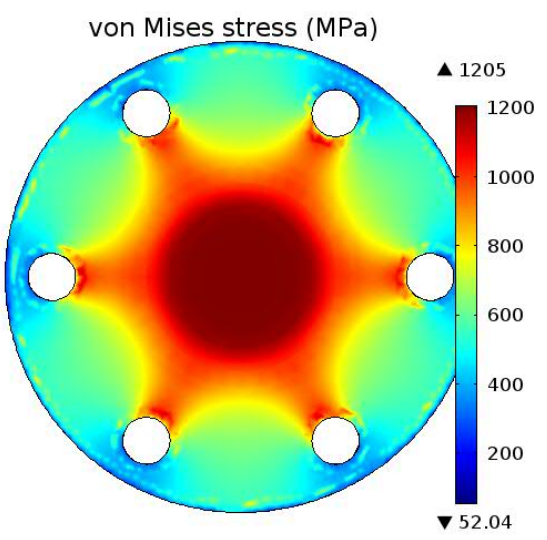

(a)

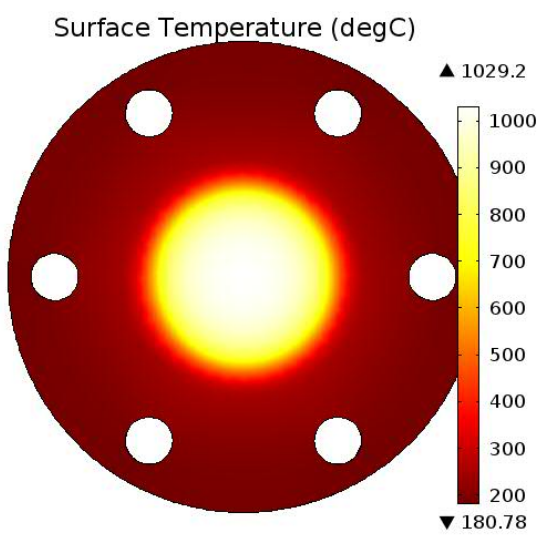

(b)

Figure 7: Surface plots at $3.5 \mathrm{sec}$ of a $4 \mathrm{sec}$ plasma pulse, which was ramped up to a maximum heat flux of 20 $\mathrm{MW} / \mathrm{m}^{2}$, (a) von Mises stress on the front face of the sample, note that the stresses reach the indicated yield point of tungsten, (b) shows the corresponding temperature profile of sample, the geometry lies in the xz-plane with the $y$-coordinate axis perpendicular to the sample surface. 


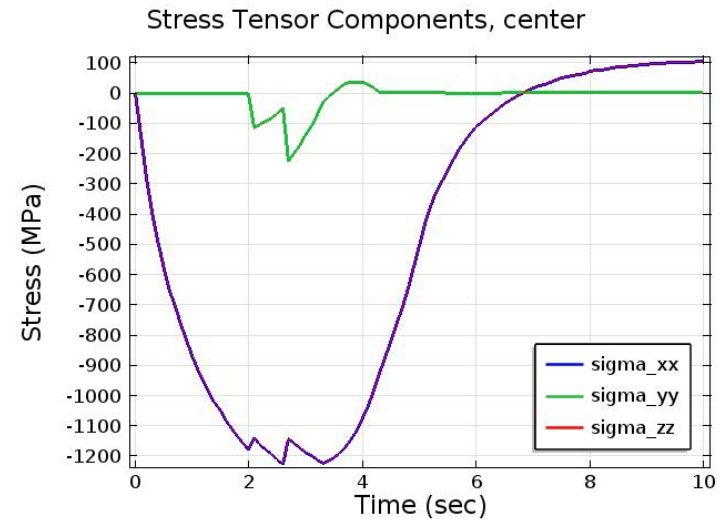

(a)

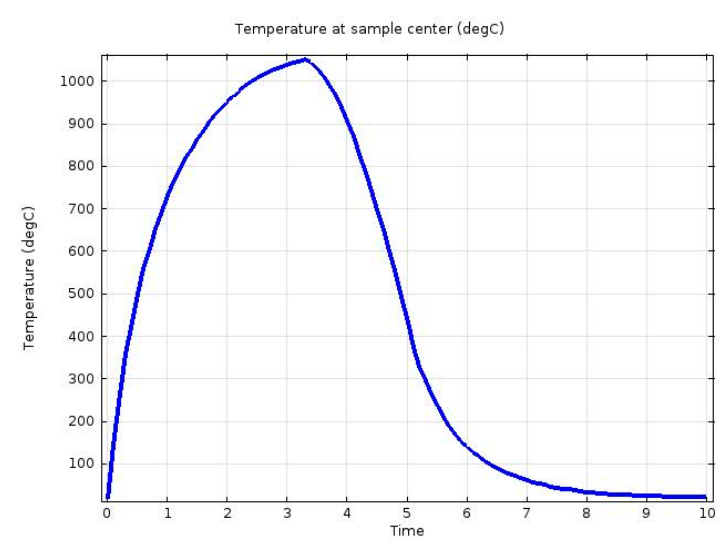

(b)

Figure 8: FEM profiles at sample center for a $4 \mathrm{sec}$ pulse ramping up to $20 \mathrm{MW} / \mathrm{m}^{2}$, (a) stress tensor components which show a residual tensile stress present in the material at the end of the thermal cycle, sigma_xx, sigma_yy, sigma_zz are the stress tensor components in the $\mathrm{x}, \mathrm{y}$, and $\mathrm{z}$ directions respectively, the sample lies in the xz-plane with the y direction normal to the surface, due to the symmetry of the sample, sigma_xx and sigma_zz are the same, and thus overlap in the figure, (b) temperature rise and fall during exposure

material restricts the contraction because it is only elastically loaded, hence resulting in a net tensile stress at the end of the cycle. This net tensile stress at the end of the thermal cycle is compounded over each plasma transient until cracking is initiated by the material in an attempt to relieve plastic strain. This behavior can be seen in Figures 6(a) and 6(b) for a sample exposed to 1000 pulses, each carrying a heat flux of $25 \mathrm{MW} / \mathrm{m}^{2}$.

\subsection{Modeling Surface Fracture}

Classical fracture mechanics (e.g. [16]), or more modern versions (e.g. XFEM [17]) modeling of plasma-induced surface damage of the type investigated here is very limited, because of the complex nature of fracture surfaces, and the high density of surface and subsurface cracks, and the intimate connection between plasticity and fracture in this situation. Fundamentally new approaches are thus needed to describe plasma-induced surface cracking and damage. Our group has begun the development of two complementary approaches to describe plasmainduced fracture. The first is based on the idea that in some regions of the surface, the density of surface cracks is so high that a distributed form of damage description is appropriate. Thus, we have developed a phase field model that can predict the extension of damage in zones in close proximity of main cracks [18]. The second approach under development is an extension of our work on representing crack surfaces as aggregates of Volterra dislocations [19, 20]. The current extension is non-trivial, because the elasto-plasticity model is to be combined with a crack nucleation and extension model in 3-D. A two-dimensional version of the model has successfully shown that this discrete representation of cracks is a viable alternative to classical fracture mechanics, and that quantities such as stress intensity factors in finite domains, J-integrals around crack tips, and the crack opening profile are all predicted well with the model [21]. The complete surface fracture model and its correlation with the present experimental observations will be reported in a future publication. 


\section{Summary and Conclusions}

The High Energy Flux Test Facility (HEFTY), was designed and constructed at UCLA, it is equipped with an $80 \mathrm{~kW}$ Praxair model SG-100 plasma gun used to simulate severe plasma transient conditions in a range of devices. The facility allows for well-instrumented testing of the effects of mild plasma transients (with heat flux $\approx<100 \mathrm{MW} / \mathrm{m}^{2}$ ), lasting a long duration of a few seconds to thousands of seconds. Testing of tungsten samples, exposed to a plasma heat fluence of up to $100 \mathrm{GJ} / \mathrm{m}^{2}$ revealed a number of interesting surface damage characteristics. The results of experimental observations are summarized below:

1. Unexposed $\mathrm{W}$ micro-pillars exhibited the tendency of high strain plastic deformation. This may be attributed to the small ratio of pillar size to grain size. This behavior is in contrast to the more brittle failure typical of bulk tungsten.

2. Mechanical compression test data indicate that the tungsten surface progressively softens with increasing exposure to the plasma heat flux, and that work hardening changes from a linear to a parabolic mode as a result of plasma exposure.

3. A delicate interplay between recovery and hardening mechanisms is expected to depend on the plasma pulse intensity, duration, and the sample cooling rate.

4. The deformation mechanism of $\mathrm{W}$ micro-pillars change from a barreling mode to a more standard shearing failure mode as a result of plasma heating.

5. Plasma transient induced surface fracture showed very complex topology, not seen in mechanically-induced fracture processes.

Results from a Finite Element (FE) thermo-elastoplasticity model show that the sample surface is under compressive stresses leading to plastic deformation during the heat up phase of the transient. This is followed by tensile surface stresses during the cool-down phase of the pulse, resulting in multi-scale fracture surfaces (from nano-meters to microns). Modeling of this complex network of surface cracks is beyond the capabilities of classical fracture mechanics. Ongoing efforts in our group focus on two main approaches: (1) a phase field approach for distributed fracture, and (2) a discrete crack surface front tracking approach, with cracks represented by Volterra dislocations.

\section{Acknowledgements}

This work is partially supported by the Air Force Office of Scientific Research (AFOSR), Award No: FA9550-11-1-0282 with UCLA, and by the US Department of Energy, Award Number DE-FG02-03ER54708 with UCLA.

\section{References}

\section{References}

[1] Gianfranco Federici, Charles H Skinner, Jeffrey N Brooks, Joseph Paul Coad, Christian Grisolia, Anthony A Haasz, Ahmed Hassanein, Volker Philipps, C Spencer Pitcher, Joachim Roth, et al. Plasma-material interactions in current tokamaks and their implications for next step fusion reactors. Nuclear Fusion, 41(12):1967, 2001. 
[2] S Pestchanyi, I Garkusha, and I Landman. Simulation of residual thermostress in tungsten after repetitive ELM-like heat loads. Fusion Engineering and Design, 86(9):1681-1684, 2011.

[3] B Bazylev, G Janeschitz, I Landman, S Pestchanyi, A Loarte, G Federici, M Merola, J Linke, A Zhitlukhin, V Podkovyrov, et al. ITER transient consequences for material damage: modelling versus experiments. Physica Scripta, 2007(T128):229, 2007.

[4] ITER Design Team. Top level project technical document (baseline document). url=http://www.reak.bme.hu/fileadmin/user-upload/felhasznalok/aszodi/KoNET/fuzioanyagok/ITER-Plant-Description-2009.pdf

[5] RA Pitts, S Carpentier, F Escourbiac, T Hirai, V Komarov, AS Kukushkin, S Lisgo, A Loarte, M Merola, R Mitteau, et al. Physics basis and design of the iter plasma-facing components. Journal of Nuclear Materials, 415(1):S957-S964, 2011.

[6] A Kirk, S Lisgo, E Nardon, T Eich, A Herrmann, A Kallenbach, and A Loarte. Physics of elm power fluxes to plasma facing components and implications for iter. Journal of Nuclear Materials, 390:727-732, 2009.

[7] John D Sethian, A Rene Raffray, Jeffery Latkowski, James P Blanchard, Lance Snead, Timothy J Renk, and Shahram Sharafat. An overview of the development of the first wall and other principal components of a laser fusion power plant. Journal of Nuclear Materials, 347(3):161-177, 2005.

[8] VI Tereshin, AN Bandura, OV Byrka, VV Chebotarev, IE Garkusha, I Landman, VA Makhlaj, IM Neklyudov, DG Solyakov, and AV Tsarenko. Application of powerful quasi-steady-state plasma accelerators for simulation of ITER transient heat loads on divertor surfaces. Plasma Physics and Controlled Fusion, 49(5A):A231, 2007.

[9] IE Garkusha, AV Burdakov, IA Ivanov, EP Kruglyakov, KN Kuklin, IS Landman, VA Makhlaj, SV Polosatkin, AA Shoshin, VI Tereshin, et al. Plasma-surface interaction during ITER transient events: Simulation with QSPA Kh-50 \& GOL-3 facilities. Problems of Atomic Science \& Technology, (6):58-60, 2008.

[10] VA Makhlaj, IE Garkusha, NN Aksenov, AA Chuvilo, MS Ladygina, I Landman, J Linke, SV Malykhin, S Pestchanyi, AT Pugachev, et al. Simulation of iter edge-localized modes' impacts on the divertor surfaces within plasma accelerators. Physica Scripta, 2011(T145):014061, 2011.

[11] William D Klopp and Walter R Witzke. Mechanical properties and recrystallization behavior of electron-beam-melted tungsten compared with arc-melted tungsten. Technical report, DTIC Document, 1966.

[12] M Pozuelo, YW Chang, and J-M Yang. In-situ microcompression study of nanostructured mg alloy micropillars. Materials Letters, 108:320-323, 2013.

[13] Ju-Young Kim, Dongchan Jang, and Julia R Greer. Tensile and compressive behavior of tungsten, molybdenum, tantalum and niobium at the nanoscale. Acta Materialia, 58(7):2355-2363, 2010. 
[14] Michael D Uchic, Paul A Shade, and Dennis M Dimiduk. Micro-compression testing of fcc metals: a selected overview of experiments and simulations. JOM, 61(3):36-41, 2009.

[15] Erik Lassner and Wolf-Dieter Schubert. Tungsten: Properties, Chemistry, Technology of the Elements, Alloys, and Chemical Compounds. Springer, 1999.

[16] JR Rice and GF Rosengren. Plane strain deformation near a crack tip in a power-law hardening material. Journal of the Mechanics and Physics of Solids, 16(1):1-12, 1968.

[17] Goangseup Zi and Ted Belytschko. New crack-tip elements for xfem and applications to cohesive cracks. International Journal for Numerical Methods in Engineering, 57(15):2221-2240, 2003.

[18] Tamer Crosby and Nasr Ghoniem. Phase-field modeling of thermomechanical damage in tungsten under severe plasma transients. Computational Mechanics, 50(2):159-168, 2012.

[19] Nasr M Ghoniem and Jianming Huang. The elastic field of general-shape 3-D cracks. Philosophical Magazine, 86(27):4195-4212, 2006.

[20] Akiyuki Takahashi and Nasr M Ghoniem. Fracture mechanics of propagating 3-D fatigue cracks with parametric dislocations. Philosophical Magazine, (ahead-of-print):1-18, 2013.

[21] Andrew Sheng, Tamer Crosby, and Nasr Ghoniem. Modeling propagating 2-D cracks in finite geometry with coupled Volterra dislocation dynamics and finite elements. Journal of the Mechanical Behavior of Materials, in preparation(to be submitted), 2014. 